\title{
Searching high order invariants in computer imagery
}

\author{
A. Berciano • H. Molina-Abril · P. Real
}

Received: 18 February 2011 / Revised: 11 November 2011 / Accepted: 12 November 2011 /

Published online: 28 March 2012

(C) Springer-Verlag 2012

\begin{abstract}
In this paper, we present a direct computational application of Homological Perturbation Theory (HPT, for short) to computer imagery. More precisely, the formulas of the $A_{\infty}$-coalgebra maps $\Delta_{2}$ and $\Delta_{3}$ using the notion of AT-model of a digital image, and the HPT technique are implemented. The method has been tested on some specific examples, showing the usefulness of this computational tool for distinguishing $3 D$ digital images.
\end{abstract}

Keywords Digital image $\cdot$ Homological perturbation theory ·

Topological invariant $\cdot A_{\infty}$-coalgebra

\section{Introduction}

In a classical way, it is common to define algebraic topology as a branch of mathematics which uses algebraic tools to study properties of topological spaces. However, over the last two decades new lines of research focusing on algorithms and applications of algebraic topology have emerged.

Among these new areas, computer imagery and pattern recognition applications have started using topological features, in order to enrich the existing methods and

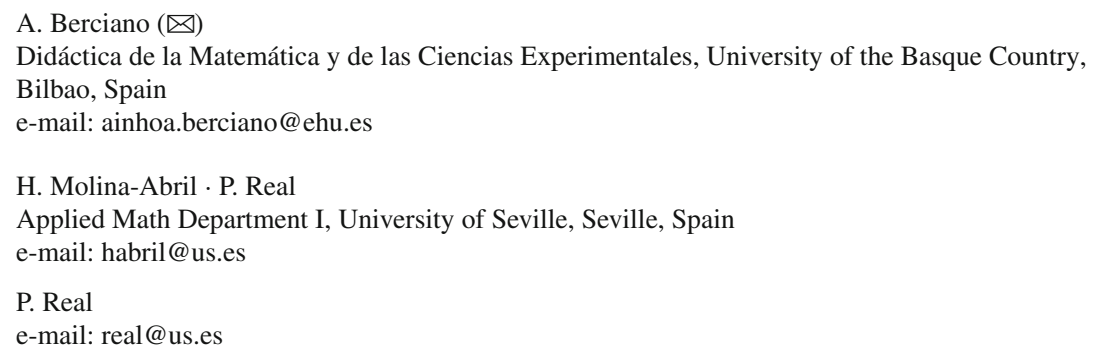


develop new ones. These applications need to face two main problems when dealing with algebraic topology: the requirement of sophisticated computations and the difficulty in obtaining explicit results.

Our purpose is to have at hand algorithms and software allowing to perform experiments and to obtain explicit results, which would be an useful tool in the context of digital imagery.

Different computer programs implementing algebraic homological procedures have been lately developed. For instance, the Kenzo system ([6]) can be used to study highly sophisticated algebraic structures coming from iterated loop spaces, using symbolic computation. Some others dealing with images and numerical computation, exclusively compute homology groups and Betti numbers (see for example [4,5]).

In order to go further, the first candidates for maps of higher complexity on homology are those extracted from an $A_{\infty}$-coalgebra structure ([12]), which is a differential graded module $M$ endowed with an infinite family of morphisms $\left(\Delta_{1}, \Delta_{2}, \Delta_{3}, \ldots,\right)$ satisfying some relationships.

In this paper we present a software that computes some explicit structural components of an $A_{\infty}$-(co)algebra deduced from a contraction, which is a special type of homotopy equivalence. This new tool enables researchers to experiment with these components, providing a base to support new research lines in computational algebraic topology and image analysis.

The paper is organized as follows: in Sect. 2 we review the preliminaries and establish our notation. The software tool is presented in Sect. 3. In Sect. 4, initial experiments performed on $3 D$ digital images are shown, with the results given by the maps $\Delta_{2}$ and $\Delta_{3}$. In the last section we discuss and comment on future work.

\section{Preliminaries}

In this section we will introduce the required concepts we need for the following sections. First, we will start introducing some algebraic aspects, to later on, continue with some topological notions.

Throughout this paper $\Lambda$ will be a commutative ring and, in the examples, we will set $\Lambda=\mathbb{Z}_{2}$. All the some basic definitions contained in the following paragraphs can be found in $([14,17])$.

A differential graded module (DG-module) is a graded module $M$ together with a square zero morphism $d: M \rightarrow M$ of degree -1 .

Given a DG-module $\left(M, d_{M}\right)$, the suspension of $M$ is the DG-module $\left(s M, d_{s M}\right)$, where $(s M)_{n}=M_{n-1}$ and $d_{s M}=-d_{M}$. Dually, the desuspension of $M$ is the DG-module $\left(s^{-1} M, d_{s^{-1} M}\right)$ given by $\left(s^{-1} M\right)_{n}=M_{n+1}$ with differential $-d_{M}$. A morphism of degree $i$ of graded modules $f: M \rightarrow N$ induces a morphism of suspensions $s f: s M \rightarrow s N$ defined by $s f=(-1)^{i} f$ and dually of desuspensions $s^{-1} f: s^{-1} M \rightarrow s^{-1} N$. The tensor module of $M$ is the differential graded module $T M=\bigoplus_{n \geq 0} M^{\otimes n}$ whose (tensor) differential $d_{t}$ is the linear extension of $d$. A morphism $f: M \rightarrow N$ of DG-modules induces a morphism $T f: T M \rightarrow T N$ via $\left.T f\right|_{M^{\otimes n}}=f^{\otimes n}$.

A differential graded coalgebra (DGC) is a DG-module $\left(C, d_{C}\right)$ endowed with a coassociative coproduct $\Delta$ and a counit $\varepsilon$ such that $d_{C}$ is a coderivation of $\Delta$ [14]. 
Given a simply connected DGC, $C$, the reduced cobar construction of $C$ is the differential graded algebra (DGA) $\bar{\Omega}(C)=T s^{-1}(C)$ with the concatenation product and differential $d_{\Omega}=d_{t}+d_{c}$, where $d_{t}$ is as above and

$$
d_{c}=\sum_{i=1}^{n}(-1)^{i-1} 1^{\otimes i-1} \otimes \Delta \otimes 1^{\otimes n-i}
$$

An $A_{\infty}$-coalgebra is a graded module $M$ together with a family of maps $\Delta_{i}: M \rightarrow$ $M^{\otimes i}$ of degree $i-2$ such that for all $i \geq 1$

$$
\sum_{n=1}^{i} \sum_{k=0}^{i-n}(-1)^{n+k+n k}\left(1^{\otimes i-n-k} \otimes \Delta_{n} \otimes 1^{k}\right) \Delta_{i-n+1}=0 .
$$

To see how is possible to compute $A_{\infty}$-coalgebras, it is required to introduce two important tools of homological algebra: (1) the notion of contraction, which is a special type of homotopy equivalence between differential graded modules and (2) the homological perturbation theory, that is a particularly useful way to obtain relatively small differential modules representing a given chain homotopy type (see [3]).

More explicitly, a contraction $c:\{N, M, f, g, \phi\}$ between the DG-modules $\left(N, d_{N}\right)$ and $\left(M, d_{M}\right)$ consists in a triple of morphisms $\{f, g, \phi\}$ where $f: N \rightarrow M$ and $g: M \rightarrow N$ are of degree zero and $\phi: N \rightarrow N$ is a homotopy operator of degree +1 that satisfy the following conditions:

$$
f g=1_{M}, \phi d_{N}+d_{N} \phi+g f=1_{N} f \phi=0, \phi g=0, \phi \phi=0 .
$$

A contraction $c:\{N, M, f, g, \phi\}$ between DG-modules induces the following contractions of desuspensions and tensor modules $[9,10]$ :

- The desuspension contraction of $c, s^{-1} c:\left\{s^{-1} N, s^{-1} M, s^{-1} f, s^{-1} g, s^{-1} \phi\right\}$.

- The tensor module contraction, $T(c)$ : $\{T(N), T(M), T(f), T(g), T(\phi)\}$, where

$$
\left.T(\phi)\right|_{T^{n}(N)}=\phi^{[\otimes n]}=\sum_{i=0}^{n-1} 1^{\otimes i} \otimes \phi \otimes(g f)^{\otimes n-i-1} .
$$

A morphism of graded modules $f: N \rightarrow N$ is called pointwise nilpotent whenever for all $x \in N, x \neq 0$, there exists a positive integer $n$ such that $f^{n}(x)=0$. A perturbation of a DG-module $N$ consists in a morphism of graded modules $\delta: N \rightarrow N$ of degree -1 , such that $\left(d_{N}+\delta\right)^{2}=0$.

In the framework of homological perturbation theory, the Basic Perturbation Lemma (BPL) [3] is an algorithm whose input is a contraction $c:\{N, M, f, g, \phi\}$ and a perturbation datum $\delta$ of $c$ and whose output is a new contraction $c_{\delta}$. The only requirement is that the composition $\phi \delta$ must be pointwise nilpotent so that the sums involved in the formulas are finite for each element of $N$ : 


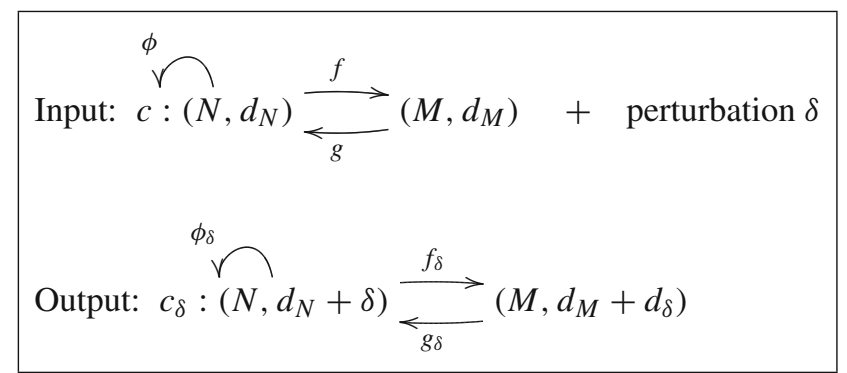

where $f_{\delta}, g_{\delta}, \phi_{\delta}, d_{\delta}$ are given by the formulas:

$$
\begin{gathered}
d_{\delta}=f \delta \sum_{i \geq 0}(-1)^{i}(\phi \delta)^{i} g ; \quad f_{\delta}=f\left(1-\delta \sum_{i \geq 0}(-1)^{i}(\phi \delta)^{i} \phi\right) \\
g_{\delta}=\sum_{i \geq 0}(-1)^{i}(\phi \delta)^{i} g ; \quad \phi_{\delta}=\sum_{i \geq 0}(-1)^{i}(\phi \delta)^{i} \phi .
\end{gathered}
$$

Taking into account the previous conditions, perturbation theory gives a theoretic algorithm for computing the structural components of an $A_{\infty}$-coalgebra (see $[1,2,10$, 11]).

The procedure by which one obtains an $A_{\infty}$-coalgebra structure on a small DG-module of a contraction, known as Tensor Trick (see [10]), follows these steps:

- As initial data, let $C$ be a DGC and $c:\{C, M, f, g, \phi\}$ be a contraction.

- Form the tensor module contraction on the desuspension

$$
T s^{-1}(c):\left\{T s^{-1}(C), T s^{-1}(M), T s^{-1}(f), T s^{-1}(g), T s^{-1}(\phi)\right\} .
$$

- Using the cosimplicial differential as a perturbation data of $T s^{-1}(c)$, apply the BPL and obtain a new contraction

$$
c b(c):\{\bar{\Omega}(C), \widetilde{\Omega}(M), c b(f), c b(g), c b(\phi)\}
$$

where $\widetilde{\Omega}(M)=T s^{-1}(M)$ with the differential $d_{T s^{-1}}+d_{\delta}$.

- Finally, the induced $A_{\infty}$-coalgebra structure on $M$ can be extracted from the tilde cobar differential $d_{\delta}$ on $\widetilde{\Omega}(M)$ and, for $i \geq 2$, its explicit formula is given by (see [11]):

$$
\Delta_{i}=(-1)^{[i / 2]+i+1} f^{\otimes i} \Delta^{[i]} \phi^{[\otimes(i-1)]} \cdots \phi^{[\otimes 2]} \Delta^{[2]} g,
$$

where

$$
\Delta^{[k]}=\sum_{i=0}^{k-2}(-1)^{i} 1^{\otimes i} \otimes \Delta \otimes 1^{\otimes k-i-2} .
$$

These maps $\Delta_{i}: M \rightarrow M^{\otimes i}$, of degree $i-2$, will be the candidates to obtain a new invariant which allows us to distinguish different images in a computational way. More precisely, to start with this family of maps, our point of view will be to focus 
on the explicit formulas of $\Delta_{2}$ and $\Delta_{3}$ acting on the representative generator(s) of the second group of the Homology of the digital images we want to study.

But, for this purpose, we need to introduce more definitions related with topology, more concretely, the notion of simplicial set, $K$, appears; where $\partial_{i}$ will be the face operators of it (see [15]).

Given a simplicial set $K$, a $q$-chain is a formal sum of cells of dimension $q$ of $K$, $K(q)$. The $q$-chains form the $q$ th chain group of $K$, denoted by $C_{q}(K)$ The boundary of a $q$-cell, $d_{q}$, is an alternate formal sum of the $(q-1)$-cells belonging to it. By linearity, $d_{q}$ can be extended to $q$-chains. The collection of boundary operators connect the chain groups $C_{q}(K)$ into the simplicial complex $\mathcal{C}(K): \ldots \stackrel{d_{2}}{\longrightarrow} C_{1}(K) \stackrel{d_{1}}{\rightarrow}$ $C_{0}(K) \stackrel{d_{0}}{\rightarrow} 0$.

Furthermore, the simplicial complex $C(K)$ of a simplicial set $K$, has a coalgebra structure defined by the Alexander Whitney diagonal $A W: C(K) \rightarrow C(K) \otimes C(K)$ defined over the simplices as

$$
A W(s)=\sum_{i=0}^{n} \partial_{i+1} \cdots \partial_{n} s \otimes \partial_{0} \cdots \partial_{i-1} s,
$$

where $\partial_{i}$ are the face operators acting on the simplex $s$ for all simplex (see [13]).

In a more general framework, a chain complex $\mathcal{C}$ is a sequence of abelian groups $C_{q}$ connected by homomorphisms $d_{q}$, such that for all $q, d_{q} d_{q+1}=0$ (a particular example of a DG-module). The set of all the homomorphisms $d_{q}$ is called the differential of $\mathcal{C}$. A $q$-chain $a \in C_{q}$ is called a $q$-cycle if $d_{q}(a)=0$. If $a=d_{q+1}\left(a^{\prime}\right)$ for some $a^{\prime} \in C_{q+1}$ then $a$ is called a $q$-boundary. Denote the groups of $q$-cycles and $q$-boundaries by $Z_{q}$ and $B_{q}$ respectively. We say that $a$ is a representative $q-$ cycle of a homology generator $\alpha$ if $\alpha=a+B_{q}$. Define the $q$ th homology group, $H_{q}(C)$, to be the quotient group $Z_{q} / B_{q}$, denoted by $H_{q}(\mathcal{C})$. The $q$ th Betti number $\beta_{q}$ is the rank of $H_{q}(\mathcal{C})$. Intuitively, given for instance a 3D image, $\beta_{0}$ is the number of components of connected pieces, $\beta_{1}$ is the number of independent "holes" and $\beta_{2}$ is the number of "cavities".

Finally, the last definition we need is the notion of $\{$ AT-model $\}$ : an AT-model ([7]) for a cell complex $K$ is an algebraic set $(K, h, f, g, \phi)$, where:

- $K$ is the cell complex.

- $h$ is a set of generators of a chain complex $\mathcal{H}$ isomorphic to the homology of $K$.

- $\{f, g, \phi\}$ is a contraction from $C(K)$ to $\mathcal{H}$.

So, summarizing, taking into account all the information above, if we have a cell complex $K$, and we are able to construct the AT-model associated to it, we will be able to induce an $A_{\infty}$-coalgebra structure on its homology using the BPL and the natural coalgebra structure of $C(K)$. So theoretically, it is possible to compute the maps of this structure, and in this paper we present an explicit algorithm which computes the first two maps: $\Delta_{2}$ and $\Delta_{3}$. 


\section{Algorithm for computing the $\Delta$ maps}

In this section an algorithm to compute the previously described maps is presented. Let us recall that the aim of this project is to analyze binary digital images within an algebraic topological framework. Homology groups and Betti numbers are a powerful tool used to differentiate digital object. But there exist multiple examples, where this information is not enough to distinguish them.

In order to progress in the analysis of digital objects by using topological information, we develop here an algorithm to compute the maps $\Delta_{2}$ and $\Delta_{3}$ acting on the representative generator(s) of the digital images. The algorithm has been implemented and some examples are shown.

The pseudocode of the algorithm is the following:

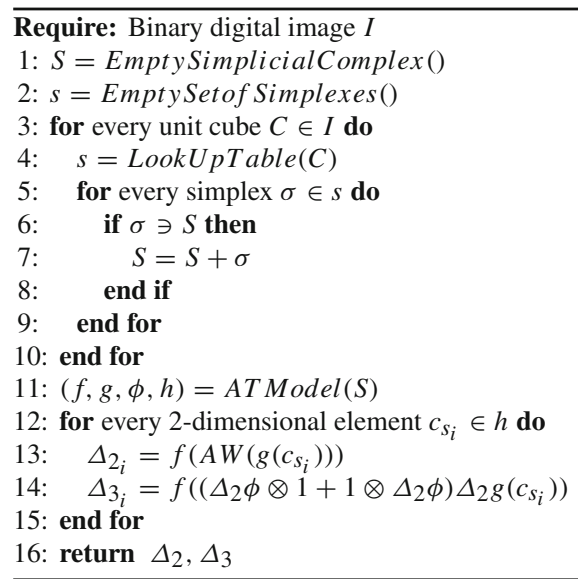

Given a binary digital image, the algorithm involves three steps:

(i) First, the simplicial complex $K$ associated to the input image is built.

(ii) Then, we obtain the AT-model for the complex $K$.

(iii) Finally, the $\Delta$ maps on the representative homology generators are computed.

\subsection{Step 1: Building the simplicial complex}

We use the algorithm proposed in [16] to construct the simplicial complex associated to the input image.

Given a binary raster image, the method subdivides the matrix into unit cubes $(2 \times 2 \times 2$ for a $3 D$ image $)$. A $3 D$-tetrahedrization of any configuration of mutually 26 -adjacent black voxels is stored in a look-up table. The union of such configurations provides a coherent simplicial complex at global level (see Fig. 1). 
(a)

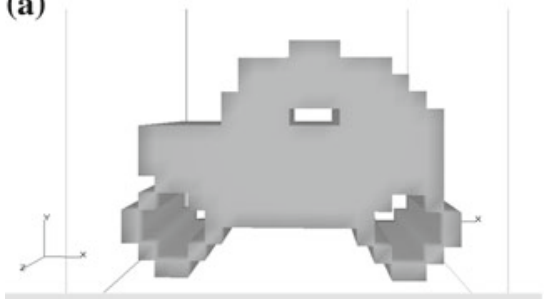

(b)

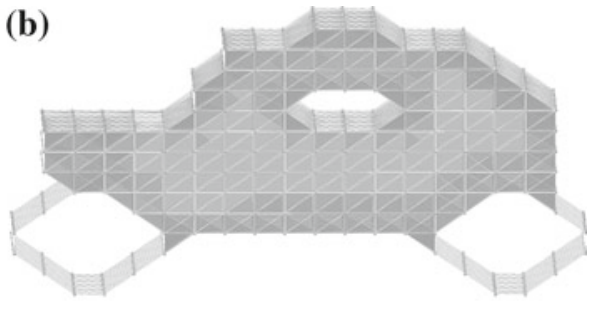

Fig. 1 a A 3D binary digital image and $\mathbf{b}$ the corresponding simplicial complex

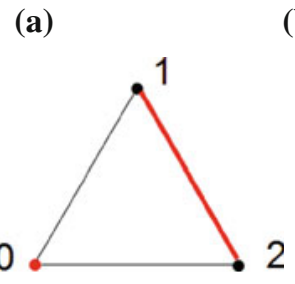

(b) \begin{tabular}{c|ccc}
$K$ & $h$ & $f$ & $\phi$ \\
\hline$\langle 0\rangle$ & $\langle 0\rangle$ & $\langle 0\rangle$ & 0 \\
$\langle 1\rangle$ & & $\langle 0\rangle$ & $\langle 0,1\rangle$ \\
$\langle 2\rangle$ & & $\langle 0\rangle$ & $\langle 0,2\rangle$ \\
$\langle 0,1\rangle$ & & & 0 \\
$\langle 0,2\rangle$ & & & 0 \\
$\langle 1,2\rangle$ & $\langle 1,2\rangle$ & $\langle 1,2\rangle$ & 0
\end{tabular}

Fig. 2 a A simplicial complex $K$ and $\mathbf{b}$ one possible AT-model for $K$ (where $g$ is the inclusion map). The elements in $h$ are colored in red. (Color figure online)

\subsection{Step 2: Obtaining the AT-model}

As we defined before, we need to work with AT-models. An example of AT-model for a simple complex is shown in Fig. 2.

The AT-model for the previously obtained simplicial complex is computed using the algorithm described in [8] (see Fig. 3). The input of the algorithm is a sorted set $\left\{\sigma_{1}, \ldots, \sigma_{m}\right\}$ of all the simplexes of $K$. Then a set of generators $h$, and a chain contraction $(f, g, \phi)$ of $C(K)$ to $\mathcal{H}$ are computed. Initially, $h$ is empty. At the $i$ th step of the algorithm, the simplex $\sigma_{i}$ is added to the subcomplex $\left\{\sigma_{1}, \ldots, \sigma_{i-1}\right\}$ and a homology class is created or destroyed. If $f\left(\partial\left(\sigma_{i}\right)\right)=0$ then $\sigma_{i}$ "creates" a homology class. Otherwise, $\sigma_{i}$ "destroys" one homology class "involved" in the expression of $f\left(\partial\left(\sigma_{i}\right)\right)$. The time complexity of the algorithm is at most $O\left(\mathrm{~m}^{3}\right)$.

\subsection{Step 3: Computing the $\Delta$ maps}

The maps $\Delta_{2}$ and $\Delta_{3}$ are computed following the formulas presented in Sect. 2.

Let $h=\left\{c_{1}, \ldots, c_{r}\right\}$ and $(f, g, \phi)$ be the output of the AT-model Algorithm applied to $K$. Let $\left\{c_{s_{1}}, \ldots, c_{s_{t}}\right\}$ be the 2-dimensional elements of $h$. If this set is empty then there are no cavities for which we can compute the $\Delta$ maps. Otherwise, the representative 2-cycles in $K$ that correspond to the generators of $H_{2}(K)$ are computed using $g$. In particular, each $g\left(c_{s_{i}}\right)=\sum \bar{c}_{i j}$ is a sum of elementary 2-simplexes enclosing at least one cavity in $K$.

Taking into account the explicit formula of the Alexander Whitney diagonal defined before, we apply it over the 2-simplexes, obtaining a sum of tensor products $A W\left(\bar{c}_{i j}\right)=$ 


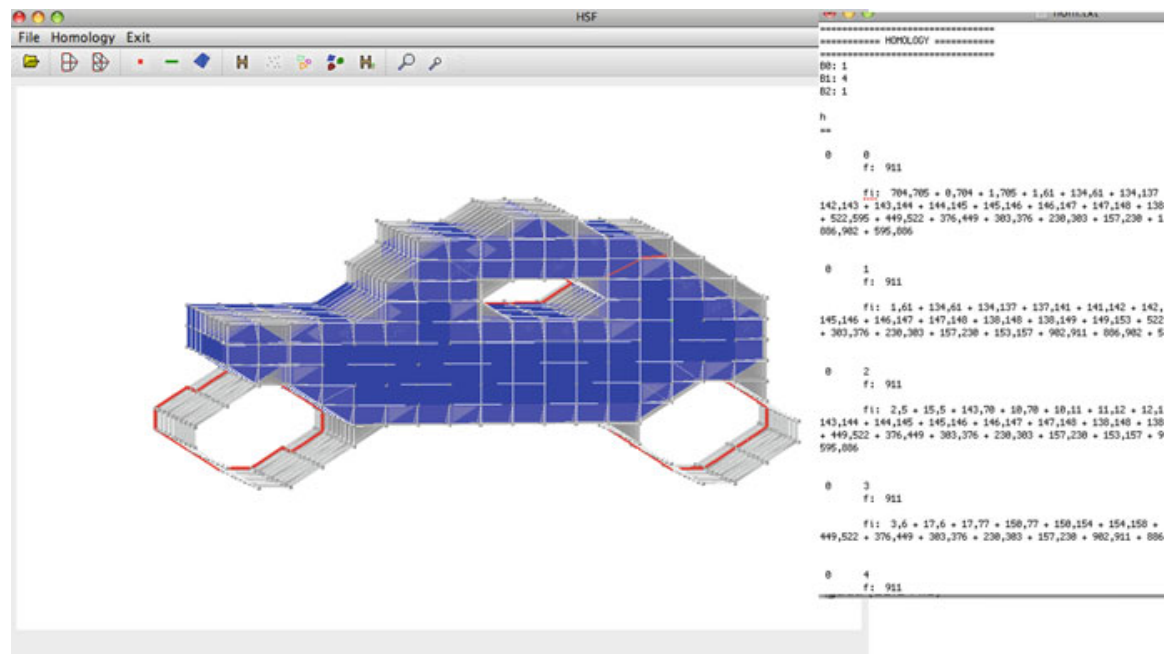

Fig. 3 The output of the homology computation process: representative homology generators are colored in red (1-cycles) and blue (2-cycles), and an output file containing the Betti numbers and the value of the morphisms $f, g$ and $\phi$ for every cell. (Color figure online)
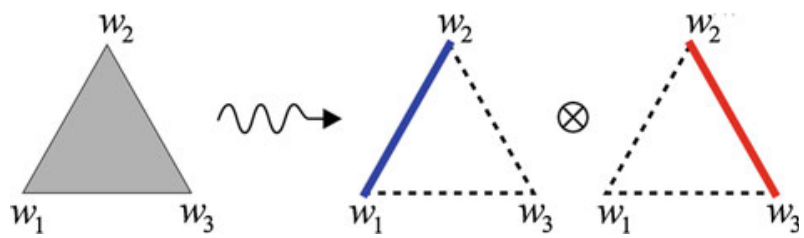

Fig. 4 The "non-trivial" part of the AW applied to a triangle as a 2-simplex

Table 1 Computations for the simplicial complex of a torus where $\eta$ represents the homology generator in $\mathcal{H}_{0}, \alpha_{1}$ and $\alpha_{2}$ the two homology generators in $\mathcal{H}_{1}$, and $\gamma$ the homology generator in $\mathcal{H}_{2}$

Representative homology generators for the complex "torus":

$\mathcal{H}_{0}=\{\eta\}, \mathcal{H}_{1}=\left\{\alpha_{1}, \alpha_{2}\right\}, \mathcal{H}_{2}=\{\gamma\}$

$\Delta_{2}$ and $\Delta_{3}$ on the representative generator of the second group:

$\Delta_{2}(\gamma)=(\eta \otimes \gamma)+(\gamma \otimes \eta)+\left(\alpha_{1} \otimes \alpha_{2}\right)+\left(\alpha_{2} \otimes \alpha_{1}\right)$

$\Delta_{3}(\gamma)=\left(\alpha_{2} \otimes \alpha_{1} \otimes \alpha_{2}\right)+\left(\alpha_{1} \otimes \alpha_{2} \otimes \alpha_{2}\right)+\left(\alpha_{2} \otimes \alpha_{1} \otimes \alpha_{1}\right)+\left(\alpha_{1} \otimes \alpha_{2} \otimes \alpha_{1}\right)$

$\sum a_{i j} \otimes b_{i j}$, where each $a_{i j}$ and $b_{i j}$ is a simplex of dimension less or equal to 2. But, with the purpose to obtain something non trivial, we are only concerned with the sum of the tensor products where the dimension of both $a_{i j}$ and $b_{i j}$ is equal to 1 (Fig. 4).

Finally, we apply the map $f$ to these pairs of chains, and in this way we obtain a description of the $\Delta_{2}$ in terms of 1-dimensional elements of $h$.

Similar procedure is performed to compute the $\Delta_{3}$ map. An example of the computations for the simplicial complex of a torus is shown in Table 1 . 
Table 2 Running time in milliseconds of the computation of $\Delta_{3}$ on the $\mathrm{H}_{2}$ generators of different simplicial complexes

\begin{tabular}{llc}
\hline Complex & Number of simplexes & Time $(\mathrm{ms})$ \\
\hline Sphere & 2,834 & 85 \\
Torus & 3,856 & 2,349 \\
2-genus torus & 6,541 & 6,478 \\
\hline
\end{tabular}

(b)

(a)
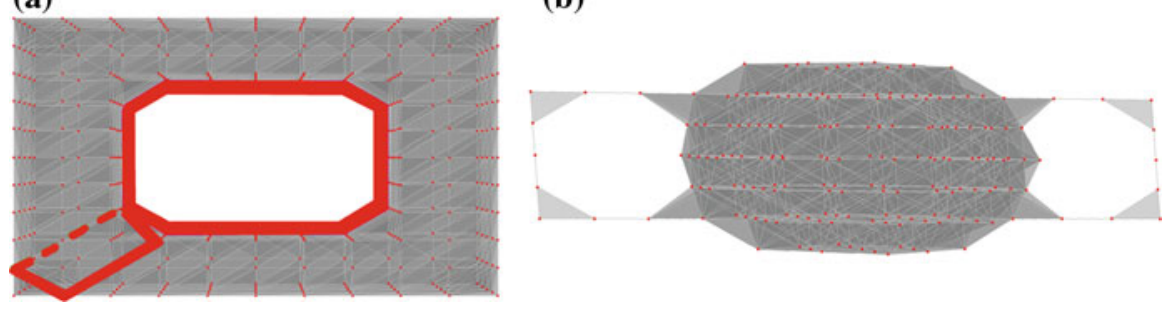

Fig. 5 a A torus of genus 2 and $\mathbf{b}$ a sphere with 2 edges

Table 3 Representative homology generators of the simplicial complexes in Fig. 5a, b

\begin{tabular}{llll}
\hline Complex & $\mathcal{H}_{0}$ & $\mathcal{H}_{1}$ & $\mathcal{H}_{2}$ \\
\hline Torus of genus 2 & $\{\eta\}$ & $\left\{\alpha_{1}, \alpha_{2}\right\}$ & $\{\gamma\}$ \\
Sphere with two edges & $\{\eta\}$ & $\left\{\alpha_{1}, \alpha_{2}\right\}$ & $\{\gamma\}$ \\
\hline
\end{tabular}

\section{Implementation and examples}

For optimal effectiveness and flexibility, the $\mathrm{C}++$ programming language has been used for the implementation. The computations are nowadays restricted to $\mathbb{Z}_{2}$ coefficients.

The input of the program is a set of $2 D$ binary images that compose the volume we want to study. Several file types are accepted (.jpg, .png, .bmp, ...).

The first step consists of acquiring the simplicial complex $K$ associated to the input image. Each black pixel in the image, will correspond to a 0 -simplex of the final complex. Then a list of simplexes and their boundaries is obtained.

In the second step, the value of $f, g$ and $\phi$ for each simplex of the complex are computed. A list of generators is stored in $h$.

Then for each element in $h$, the maps $\Delta_{2}$ and $\Delta_{3}$ are computed. Either an empty list is obtained, or a list containing a sum of tensor products of elements in $h$.

Some examples of the running time in a Intel Core i5 CPU 760, $2.8 \mathrm{GHz}, 8 \mathrm{~Gb}$ RAM, are shown in Table 2.

We show now some examples where these maps are useful for image comparison.

Example 1 A torus of genus 2 and an sphere with 2 edges (see Fig. 5 and Table 3).

Using the information in Table 3, that are the Betti numbers corresponding to the two complexes in Fig. 5, we are not able to distinguish between them. Both examples have one generator in $\mathcal{H}_{0}$, two in $\mathcal{H}_{1}$ and one in $\mathcal{H}_{2}$.

We compute now the morphisms $\Delta_{2}$ and $\Delta_{3}$ (see Table 4). 
Table $4 \Delta_{2}$ and $\Delta_{3}$ computed on the $H_{2}$ generators of Fig. 5a, b

\begin{tabular}{llr}
\hline & $\Delta_{2}$ & $\Delta_{3}$ \\
\hline$(a) \gamma$ & $(\eta \otimes \gamma)+(\gamma \otimes \eta)+\left(\alpha_{1} \otimes \alpha_{2}\right)$ & $\left(\alpha_{2} \otimes \alpha_{1} \otimes \alpha_{2}\right)+\left(\alpha_{1} \otimes \alpha_{2} \otimes \alpha_{2}\right)$ \\
& $+\left(\alpha_{2} \otimes \alpha_{1}\right)$ & $+\left(\alpha_{2} \otimes \alpha_{1} \otimes \alpha_{1}\right)+\left(\alpha_{1} \otimes \alpha_{2} \otimes \alpha_{1}\right)$ \\
$(b) \gamma$ & $(\eta \otimes \gamma)+(\gamma \otimes \eta)$ & 0 \\
\hline
\end{tabular}

(a)

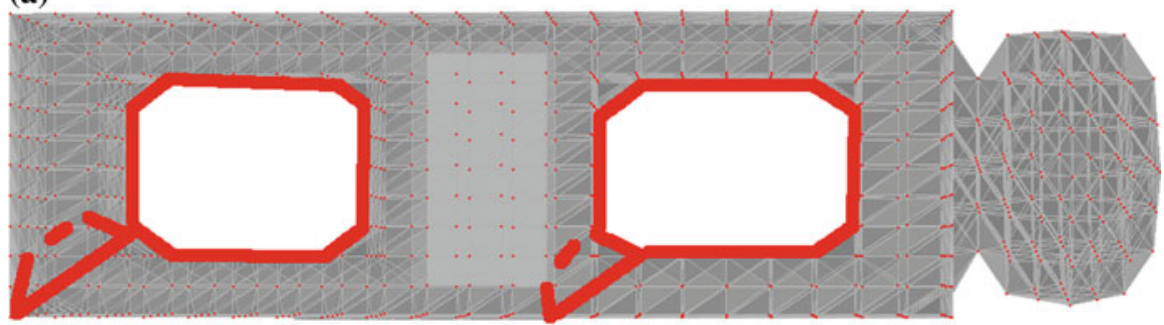

(b)

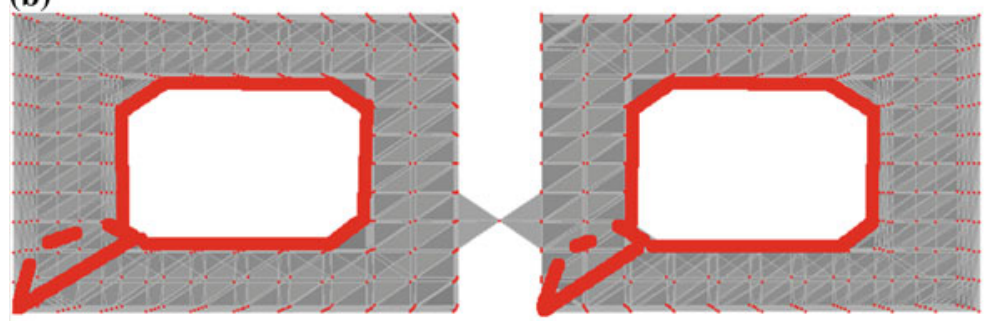

Fig. 6 a A torus of genus 2 with an attached sphere, and $\mathbf{b}$ two connected torus

Table 5 Representative homology generators of the simplicial complexes in Fig. 6a, b

\begin{tabular}{llll}
\hline Complex & $\mathcal{H}_{0}$ & $\mathcal{H}_{1}$ & $\mathcal{H}_{2}$ \\
\hline $\begin{array}{c}\text { Torus of genus 2 with } \\
\text { an attached sphere }\end{array}$ & $\{\eta\}$ & $\left\{\alpha_{1}, \alpha_{2}, \alpha_{3}, \alpha_{4}\right\}$ & $\left\{\gamma_{1}, \gamma_{2}\right\}$ \\
$\begin{array}{l}\text { Two connected torus } \\
\text { ywo }\end{array}$ & $\{\eta\}$ & $\left\{\alpha_{1}, \alpha_{2}, \alpha_{3}, \alpha_{4}\right\}$ & $\left\{\gamma_{1}, \gamma_{2}\right\}$ \\
\hline
\end{tabular}

In Table $4, \Delta_{3}$ expresses the geometrical relation between the generators of dimension one and the generators of dimension two of Fig. 5. It is clear that in the case of the torus (Fig. 5a), the cavity of dimension two can be generated by the two generators of dimension one, but this is not possible in the case of the sphere with two edges (Fig. 5b).

Example 2 A torus of genus 2 with an attached sphere, and two connected torus (see Fig. 6 and Table 5).

As we can see in Table 5, Betti numbers are the same for both images in Fig. 6. In Table 6 , the morphisms $\Delta_{2}$ and $\Delta_{3}$ are shown.

From a geometrical point of view, the results given by the morphisms $\Delta_{2}$ and $\Delta_{3}$ can be explained taking into account the way to generate the 2-cycle of the objects using a representative of the cavities of dimension one. That is, in Fig. 6 a the cavity of dimension one of the torus of genus $2\left(\gamma_{1}\right)$ can be generated as a revolution surface 
Table $6 \Delta_{2}$ and $\Delta_{3}$ computed on the $H_{2}$ generators of Figs. 6a, 5 b

\begin{tabular}{llr}
\hline & $\Delta_{2}$ & $\Delta_{3}$ \\
\hline$(a) \gamma_{1}$ & $\left(\eta \otimes \gamma_{1}\right)+\left(\gamma_{1} \otimes \eta\right)+\left(\alpha_{1} \otimes \alpha_{2}\right)$ & $\left(\alpha_{1} \otimes \alpha_{1} \otimes \alpha_{2}\right)+\left(\alpha_{1} \otimes \alpha_{2} \otimes \alpha_{1}\right)$ \\
& $+\left(\alpha_{2} \otimes \alpha_{1}\right)+\left(\alpha_{3} \otimes \alpha_{4}\right)+\left(\alpha_{4} \otimes \alpha_{3}\right)$ & $+\left(\alpha_{1} \otimes \alpha_{2} \otimes \alpha_{2}\right)+\left(\alpha_{2} \otimes \alpha_{1} \otimes \alpha_{1}\right)$ \\
& & $+\left(\alpha_{3} \otimes \alpha_{3} \otimes \alpha_{4}\right)+\left(\alpha_{3} \otimes \alpha_{4} \otimes \alpha_{3}\right)$ \\
& & $+\left(\alpha_{4} \otimes \alpha_{1} \otimes \alpha_{2}\right)+\left(\alpha_{4} \otimes \alpha_{2} \otimes \alpha_{1}\right)$ \\
& & $+\left(\alpha_{4} \otimes \alpha_{2} \otimes \alpha_{2}\right)+\left(\alpha_{4} \otimes \alpha_{3} \otimes \alpha_{3}\right)$ \\
& & $+\left(\alpha_{4} \otimes \alpha_{3} \otimes \alpha_{4}\right)$ \\
$($ a $) \gamma_{2}$ & $\left(\eta \otimes \gamma_{2}\right)+\left(\gamma_{2} \otimes \eta\right)$ & \\
$(b) \gamma_{1}$ & $\left(\eta \otimes \gamma_{1}\right)+\left(\gamma_{1} \otimes \eta\right)+\left(\alpha_{1} \otimes \alpha_{2}\right)$ & $\left(\alpha_{1} \otimes \alpha_{1} \otimes \alpha_{2}\right)+\left(\alpha_{1} \otimes \alpha_{2} \otimes \alpha_{1}\right)$ \\
& $+\left(\alpha_{2} \otimes \alpha_{1}\right)$ & $+\left(\alpha_{2} \otimes \alpha_{1} \otimes \alpha_{1}\right)+\left(\alpha_{2} \otimes \alpha_{1} \otimes \alpha_{2}\right)$ \\
(b) $\gamma_{2}$ & $\left(\eta \otimes \gamma_{2}\right)+\left(\gamma_{2} \otimes \eta\right)+\left(\alpha_{4} \otimes \alpha_{3}\right)$ & $\left(\alpha_{4} \otimes \alpha_{3} \otimes \alpha_{3}\right)+\left(\alpha_{4} \otimes \alpha_{3} \otimes \alpha_{4}\right)$ \\
& $+\left(\alpha_{3} \otimes \alpha_{4}\right)$ & $+\left(\alpha_{3} \otimes \alpha_{3} \otimes \alpha_{4}\right)+\left(\alpha_{3} \otimes \alpha_{4} \otimes \alpha_{3}\right)$ \\
\hline
\end{tabular}

of the 1-dimension cycles (generators of the torus), but the cavity of dimension two of the sphere $\left(\gamma_{2}\right)$ can not be generated in the same way. Therefore, a trivial result is obtained when $\Delta_{2}$ and $\Delta_{3}$ are computed on it. In the same way, it is simple to explain the non trivial result of $\Delta_{2}$ and $\Delta_{3}$ over the cavities of dimension two in Fig. $6 \mathrm{~b}$.

\section{Conclusions and future work}

Homology is a topological tool which does not give on its own a complete topological answer to classification, matching and recognition tasks in structural pattern recognition for 3D and 4D objects. Alexander-Whitney coproduct in homology and derived higher maps are used here for revealing new relations between homology generators in 3D binary voxel-based digital volumes. The experiments performed with the proposed homological method have demonstrated that the maps $\Delta_{2}$ and $\Delta_{3}$ allow to distinguish volumes having the same homology groups. There are some interesting questions: (a) Can we guarantee the same information of $\Delta_{2}$ and $\Delta_{3}$ for $3 \mathrm{D}$ objects working with integer coefficients?; (b) Does the map $\Delta_{3}$ provide new important information in 4D ambiance? (c) Is in fact the map $\Delta_{3}$ the one that measures the lack of associativity of $\Delta_{2}$ ? And what about the associativity of $\Delta_{3}$ ? (d) Is "acceptable" the cost involved in the computation of the maps $\Delta_{3}$ and $\Delta_{4}$ for a more general 4D digital image?

In a near future, we intend to deal with these questions as well as with homology classification tasks for 3D and 4D geometric objects based on this new technique.

\section{References}

1. Berciano, A.: A computational aproach of $a_{\infty}$-(co)algebras. Int. J. Comput. Math. 87(4), 935-953 (2010)

2. Berciano, A., Rubio, J., Sergeraert, F.: A case study of $a_{\infty}$-structures. Georgian Math. J. 17, 57-77 (2010)

3. Brown, R.: The twisted eilenberg-zilber theorem. In: In Simposio di Topologia (Messina, 1964), Edizioni Oderisi, Gubbio (1965)

4. Plex: Simplicial complexes in Matlab. http://comptop.stanford.edu/programs/plex/

5. Computational Homology Project. http://chomp.rutgers.edu/

6. Dousson, X., Rubio, J., Sergeraert, F., Siret, Y.: The kenzo program. http://www.fourier.ujfgrenoble. $\mathrm{fr} /$ sergeraert/ (1999) 
7. González-Díaz, R., Ion, A., Iglesias-Ham, M., Kropatsch, W.G.: Irregular graph pyramids and representative cocycles of cohomology generators. 7th IAPR-TC-15 Workshop on Graph-based Representations in Pattern Recognition, Venice (Italy) (2009) (to appear in LNCS)

8. González-Díaz, R., Medrano, B., Sánchez Peláez, J., Real, P.: Simplicial perturbation techniques and effective homology. CASC, LNCS 4194, 166-177 (2006)

9. Gugenheim, V.K.A.M., Lambe, L.A.: Perturbation theory in differential homological algebra I. Ill. J. Math. 33(4), 566-582 (1989)

10. Gugenheim, V.K.A.M., Lambe, L.A., Stasheff, J.D.: Perturbation theory in differential homological algebra II. Ill. J. Math. 35(3), 357-373 (1991)

11. Jimenez, M.J., Real, P.: Rectifications of $a_{\infty}$-algebras. Commun. Algebra 35(1532-4125), 2731-2743 (2007)

12. Kadeishvili, T.: On the homology theory of fibrations. Russ. Math. Surv. 35(3), 231-238 (1980)

13. Kozlov, D.: Combinatorial Algebraic Topology. Springer, Germany (2008)

14. Mac Lane, S.: Homology Classics in Mathematics. Springer, Berlin (1995)

15. May, P.: Simplicial Sets in Algebraic Topology. University of Chicago, Chicago (1967)

16. Molina-Abril, H., Real, P.: Advanced homology computation of digital volumes via cell complexes. SSPR 2008, LNCS 5342, 361-371 (2008)

17. Weibel, C.A.: An Introduction to Homological Algebra. Cambridge Studies in Advanced Mathematics 38. Cambridge University Press, Cambridge (1994) 